DESY $10-078$

ISSN $0418-9833$

May 2010

\title{
Treatment of Photon Radiation in Kinematic Fits at Future $e^{+} e^{-}$Colliders
}

\author{
M. Beckmann ${ }^{1}$, B. List ${ }^{2}$, and J. List ${ }^{1}$ \\ 1- Deutsches Elektronen-Synchrotron DESY \\ Notkestr. 85 \\ 22607 Hamburg, Germany \\ 2- University of Hamburg \\ Institute for Experimental Physics \\ Luruper Chaussee 149 \\ 22761 Hamburg, Germany
}

\begin{abstract}
Kinematic fitting, where constraints such as energy and momentum conservation are imposed on measured four-vectors of jets and leptons, is an important tool to improve the resolution in high-energy physics experiments. At future $e^{+} e^{-}$colliders, photon radiation parallel to the beam carrying away large amounts of energy and momentum will become a challenge for kinematic fitting. A photon with longitudinal momentum $p_{\mathrm{z}, \gamma}(\eta)$ is introduced, which is parametrized such that $\eta$ follows a normal distribution. In the fit, $\eta$ is treated as having a measured value of zero, which corresponds to $p_{\mathrm{z}, \gamma}=0$. As a result, fits with constraints on energy and momentum conservation converge well even in the presence of a highly energetic photon, while the resolution of fits without such a photon is retained. A fully simulated and reconstructed $e^{+} e^{-} \rightarrow q \bar{q} q \bar{q}$ event sample at $\sqrt{s}=500 \mathrm{GeV}$ is used to investigate the performance of this method under realistic conditions, as expected at the International Linear Collider.
\end{abstract}

To be submitted to Nucl. Instrum. Meth. A 


\section{Introduction}

Radiation of photons at angles so small that they escape along the beam pipe is usually not taken into account in kinematic fits. At previous $e^{+} e^{-}$colliders such as LEP, the losses due to photon radiation were acceptable [1]. At future facilities such as the International Linear Collider (ILC) [2] or the Compact Linear Collider (CLIC) [3], photon radiation will be much stronger due to higher center-of-mass energies and stronger focussing of the beams, which makes it desirable to model photon radiation in kinematic fits.

Kinematic fitting is a well-established tool to improve jet energy and invariant mass resolutions. A number of four-vectors representing the final state particles is fitted under constraints such as energy and momentum conservation. The four-vectors are parametrized by suitably chosen variables such that the measured values follow an approximately Gaussian distribution around the true values. A $\chi^{2}$ that quantifies the deviation between measured and fitted parameters is minimized under the condition that the imposed constraints are fulfilled [4].

The improvement in resolution emerges from the redundant information contained in the measured values in the presence of constraints. Unmeasured parameters reduce the redundancy, since one constraint is used up for each unmeasured parameter to determine its value. The redundancy is quantified by the number of degrees of freedom, which is given by the number of constraints minus the number of unmeasured parameters.

The two main effects that cause the emission of photons collinear with the incoming beams so that they escape the main detector are initial state radiation (ISR) and beamstrahlung. ISR is a higher-order QED effect, at which real photons are emitted before the actual interaction. Beamstrahlung is caused by the electrical fields of the bunches colliding with each other: electrons in the one bunch are deflected by the field of the other bunch and thus emit bremsstrahlung photons.

ISR is characterized by an energy spectrum that follows a power law with an exponent of roughly -0.9 [5]. Thus the vast majority of events have at most one ISR photon with an energy above a few $\mathrm{GeV}$, which is the accuracy to which the total energy and longitudinal momentum of fully hadronic events can be measured by a typical detector envisioned for the linear collider. This photon can, however, carry substantial energy of tens of GeV. Beamstrahlung on the other hand has an energy spectrum with an exponent of $-2 / 3$, but with an additional exponential suppression of high energy photons [6]. The mean number of beamstrahlung photons emitted prior to the interaction can be of order one or even larger, depending on the beam parameters.

This paper presents a novel method to take the energy and longitudinal momentum of photon radiation into account in kinematic fits. A priori information about the momentum spectrum of photon radiation is used to treat the photon's momentum as a measured parameter in the fit. As a test case, the production of $W^{+} W^{-} / Z^{0} Z^{0}$ pairs decaying to light quark jets at the ILC is considered, with fully simulated Monte Carlo events as reconstructed by the International Large Detector (ILD) [7]. A more detailed description of the method and its application tests can be found in [8].

The main focus of this method is an improved treatment of the effects of ISR, because ISR is the main source of highly energetic photons. Therefore, only a single photon is included 
in the kinematic fit, with an energy spectrum given by a power law, as expected for ISR. A similar method with the inclusion of two photons in the fit and an energy spectrum describing the combined effects of ISR and beamstrahlung is the subject of ongoing work and beyond the scope of the current publication. However, the method presented here leads to a significant improvement also in the presence of beamstrahlung, as shown in section 3 .

\section{Representation of the photon}

Since photons from ISR and beamstrahlung escaping the detector have to a good approximation zero transverse momentum with respect to the beam direction, they affect mainly the conservation of (detected) energy $E$ and longitudinal momentum $p_{\mathrm{z}}$. The simplest method to cope with highly energetic photons in a constrained kinematic fit is therefore to drop the energy and longitudinal momentum conservation constraints, thus losing two degress of freedom.

A somewhat better solution is to introduce a fit object representing the undetected photon with one free, unmeasured parameter, namely its longitudinal momentum $p_{\mathrm{z}, \gamma}$, and set $p_{\mathrm{x}, \gamma}=$ $p_{\mathrm{y}, \gamma}=0$ and thus $E_{\gamma}=\left|p_{\mathrm{z}, \gamma}\right|$. This allows the energy and $p_{\mathrm{z}}$ constraints to be recovered, at the price of one unmeasured parameter, so that one degree of freedom is regained.

However, this approach neglects the information about the momentum spectrum of the photons. Here this information is used so that the photon is treated as a particle with a measured momentum of zero and an uncertainty derived from its known momentum spectrum.

\subsection{Parametrization of the Photon Energy}

In a kinematic $\chi^{2}$ fit the measured four-vector components of a particle or jet are parametrized with parameters $\eta_{\mathrm{i}}$ (e.g., $\left.E, \theta, \phi\right)$ such that the difference $\eta_{\mathrm{i}, \text { meas }}-\eta_{\mathrm{i}, \text { true }}$ between the measured $\eta_{\mathrm{i} \text {,meas }}$ and the true value $\eta_{\mathrm{i}, \text { true }}$ follows a Gaussian distribution with zero mean and standard deviation $\delta \eta_{\mathrm{i}}$ (for reasons of notational simplicity we limit the discussion to the case where the parameters $\eta_{\mathrm{i}}$ are uncorrelated). Then,

$$
\chi^{2}=\sum_{i} \frac{\left(\eta_{\mathrm{i}, \mathrm{meas}}-\eta_{\mathrm{i}}\right)^{2}}{\delta \eta_{\mathrm{i}}^{2}}
$$

is, apart from a constant, identical to the negative logarithm of the likelihood to obtain the measured values, given the values $\eta_{\mathrm{i}}$ :

$$
\chi^{2}=-\ln \mathcal{P}\left(\eta_{\mathrm{i}, \text { meas }} \mid \eta_{\mathrm{i}}\right)+\text { const. }
$$

Thus, the $\chi^{2}$ fit seeks the best estimate $\eta_{\mathrm{i}}$ for the true parameter values by maximizing the likelihood to get the observed parameter values $\eta_{\text {meas }}$ under the condition that the imposed constraints are fulfilled, which are expressed by a number of constraint functions $g_{\mathrm{k}}\left(\eta_{\mathrm{i}}\right)=0$. No assumption is made, or is necessary, about the distribution of the true parameter values $\eta_{\mathrm{i}, \text { true }}$ 
However, if an ensemble of events is considered where the distribution of a parameter $\eta_{\text {true }}$ is known to be Gaussian with zero mean, then for this ensemble the choice $\eta_{\text {meas }}=0$ also leads to a Gaussian distribution of $\eta_{\text {meas }}-\eta_{\text {true }}$, and for such an ensemble it is justified to estimate $\eta_{\text {true }}$ by means of a $\chi^{2}$ fit.

In the case of photon radiation, the distribution of the unmeasured momentum $p_{\mathrm{z}, \gamma}$ is known, though definitely non-Gaussian. Thus we seek a parametrization of the photon's momentum $p_{\mathrm{z}, \gamma}=p_{\mathrm{z}, \gamma}(\eta)$ such that the true value of $\eta$ follows a Gaussian distribution with mean zero and unit standard deviation $\delta \eta=1$. Then the photon will be treated as if it had a measured value of $\eta_{\text {meas }}=0$. The photon will then be added to the list of fit objects in the kinematic fit, thereby introducing an additional contribution to the overall $\chi^{2}$ of $\eta^{2} / \delta \eta^{2}=\eta^{2}$. By this procedure, the a priori knowledge of the photon's energy spectrum (in particular the fact that it is negligibly small in most cases) is used, and all energy and momentum constraints can be applied.

The probability density function $\mathcal{P}(y)$ for the energy fraction $y=E_{\gamma} / E_{\text {beam }}$ carried by initial state radiation is well approximated by [5]

$$
\mathcal{P}(y)=\beta y^{\beta-1},
$$

with the exponent $\beta$ given by

$$
\beta=\frac{2 \alpha}{\pi}\left(\ln \frac{s}{m_{\mathrm{e}}^{2}}-1\right),
$$

which corresponds to $\beta=0.1235$ for $\sqrt{s}=500 \mathrm{GeV}$.

Considering that an ISR photon can be emitted by either beam leads to

$$
\mathcal{P}\left(p_{\mathrm{z}, \gamma}\right)=\frac{\beta}{2 E_{\max }} \cdot\left|\frac{p_{\mathrm{z}, \gamma}}{E_{\max }}\right|^{\beta-1},
$$

where $E_{\text {max }} \leq E_{\text {beam }}$ is the maximum possible photon energy. As a consequence, the quantity $z$ given by

$$
z=\operatorname{sign}\left(p_{\mathrm{z}, \gamma}\right)\left(\frac{\left|p_{\mathrm{z}, \gamma}\right|}{E_{\max }}\right)^{\beta}
$$

is uniformely distributed between -1 and 1 , and hence

$$
\eta=\sqrt{2} \cdot \operatorname{erf}^{-1}(z)
$$

follows a Gaussian distribution with zero mean and unit standard deviation. Here, $\operatorname{erf}^{-1}(z)$ denotes the inverse of the error function given by erf $(x)=\frac{2}{\sqrt{\pi}} \int_{0}^{x} \mathrm{e}^{-t^{2}} \mathrm{~d} t$.

Conversely the expressions for $z$ and $p_{z, \gamma}$ as a function of the parameter $\eta$ read

$$
\begin{aligned}
z(\eta) & =\operatorname{erf}(\eta / \sqrt{2}) \\
p_{\mathrm{z}, \gamma}(\eta) & =\operatorname{sign}(z) E_{\max }|z|^{\frac{1}{\beta}} \\
& =\operatorname{sign}(\eta) E_{\max }[\operatorname{erf}(|\eta| / \sqrt{2})]^{\frac{1}{\beta}} .
\end{aligned}
$$




\subsection{Properties of the Parametrization}

Fig. 11 shows a graph of $p_{\mathrm{z}, \gamma}(\eta)$ for $E_{\max }=225 \mathrm{GeV}$ and $\beta=0.1235$. The function has four distinct bends around $|\eta| \approx 1$ and $|\eta| \approx 2.5$. It is flat around $\eta=0$, reflecting the fact that the majority of ISR photons have negligible momentum; only for $|\eta|>0.7$ significant momenta above $1 \mathrm{GeV}$ are predicted.

Around $\eta=0$ the value of $p_{z, \gamma}$ does not change and thus cannot influence the global $\chi^{2}$ of the kinematic fit. Therefore, the penalty term $\eta^{2}$ leads to a local minimum of the $\chi^{2}$ at this value of $\eta$. This will also be the global minimum if the measured four-momenta of the final state particles are compatible with no missing momentum from ISR. In this case, a fit with a photon fit object has exactly the same result as a fit without a photon.

Due to this local minimum, any minimization method based on derivatives will always yield $\eta=0$ if this value is used as starting value in the minimization. Therefore, to find the global minimum, in addition a different starting value must be tried, for instance $\eta\left(-p_{z, \text { miss }}\right)$ calculated from the missing $p_{\mathrm{z}}$ of the event.

For $1 \lesssim|\eta| \lesssim 2.5$, the curve rises steeply, so that large values of missing energy and momentum from ISR and beamstrahlung can be accomodated by the kinematic fit at a moderate penalty $\eta^{2}$. Thus, for photon momenta that are large compared to the detector resolution, the kinematic fit should find a global minimum of the $\chi^{2}$ close to the true photon momentum, with a negligible bias towards low photon energies, despite the fact that the "measured" value of $\eta$ and thus of $p_{\mathrm{z}, \gamma}$ is set to zero.

Above $|\eta| \approx 2.5$, corresponding to $\left|p_{\mathrm{z}, \gamma}\right| / E_{\max } \approx 0.9$, the curve flattens again, so that extremely large photon momenta are suppressed due to the fact that they are increasingly unlikely. This region is, however, of little interest in realistic analyses.

\section{Performance Tests}

The method described above is applied to the process $e^{+} e^{-} \rightarrow W^{+} W^{-} \rightarrow 4$ jets events. The fraction of successful fits, the width and the shift of the reconstructed $W^{ \pm}$mass peak are used to compare the performance of the various kinematic fit variants.

\subsection{Data Set}

The analysis sample was generated using the matrix element generator WHIZARD [9], which takes into account all Feynman diagrams leading to a given final state, including interference terms. Here, the process $e^{+} e^{-} \rightarrow u \bar{d} d \bar{u}$ is chosen, because it contains no heavy quarks in the final state, so that the jet energy and angle measurement is not compromised by the presence of neutrinos from semileptonic decays.

In addition to the dominant $W^{ \pm}$pair production, also $Z^{0}$ pair production contributes to the formation of this final state, and at a even smaller level single boson production with subsequent 
radiation off final state quarks. Due to the inclusion of interference effects, it is conceptually not possible to identify events from these additional processes and remove them. The centreof-mass energy is $\sqrt{s}=500 \mathrm{GeV}$, and the $W^{ \pm}$mass was set to $m_{\mathrm{W}}^{\mathrm{gen}}=80.419 \mathrm{GeV}$.

The initial state radiation is also simulated by WHIZARD, and one ISR photon per incoming lepton is stored in the event record. In contrast, the energy spread of the incoming beams and beamstrahlung is taken into account in the event generation by a corresponding variation of the momenta of the incoming leptons, using a beamstrahlung spectrum that was simulated with GUINEA-PIG [10]. For this calculation the nominal beam parameter set of the ILC was assumed, [11], in particular an energy spread of $0.14 \%$ and $0.07 \%$ for the electron and positron beams, respectively, a beamstrahlung parameter $\Upsilon_{\text {ave }}=0.047$, a mean energy loss by beamstrahlung of $\delta_{\mathrm{BS}}=0.023$, and a vertical disruption parameter of $D_{\mathrm{y}}=19.1$.

Thus, the momenta of ISR photons are directly accessible in the event record, while the combined effect of beamstrahlung and beam energy spread has to be deduced from the total four-momentum of all final state particles.

A full simulation of the ILD detector [7] is performed by the GEANT based simulation program MOKKA [12]. In the event reconstruction, which is implemented as part of the software package MarlinReco [13], the tracks are matched to the calorimeter clusters by the Pandora particle flow algorithm [14] and the resulting reconstructed particles are forced into four jets by the Durham algorithm [15]. Each of the four jets has to have a minimum energy of $E_{\text {jet }}>5 \mathrm{GeV}$ and a polar angle that fulfills $\left|\cos \theta_{\text {jet }}\right|<0.9$.

The jet momentum four-vectors are parametrized in terms of energy $E_{\text {jet }}$, polar angle $\theta$ and azimuthal angle $\phi$, with resolutions [16]:

$$
\begin{aligned}
\delta E_{\text {jet }} / E_{\text {jet }} & =32.24 \% / \sqrt{E_{\text {jet }}}+1.242 \cdot 10^{-4} E_{\text {jet }}-1.446 \% \\
\delta \theta & =0.03925 / \sqrt{E_{\text {jet }}}+0.3373 / E_{\text {jet }} \\
\delta \phi & =0.05873 / \sqrt{E_{\text {jet }}}+0.3207 / E_{\text {jet }} .
\end{aligned}
$$

Since the method presented here concerns radiation escaping the main detector, a subsample of events is selected such that at generator level only negligible energy from ISR is present in the detector acceptance. Therefore, events are rejected with ISR photons of energy $E_{\gamma}^{\text {gen }}>5 \mathrm{GeV}$ and polar angles $0.29^{\circ} \leq \theta_{\gamma}^{\text {gen }} \leq 179.71^{\circ}$, which corresponds to the acceptance of the beampipe calorimeter (BeamCAL). No cut is applied on the energy or direction of the beamstrahlung.

In order to investigate the influence of ISR and beamstrahlung on the performance of the kinematic fit, the event sample is divided into three subsamples according to the total energy $E_{\mathrm{ISR}}$ of the ISR photons:

- A subsample with small $E_{\mathrm{ISR}}<5 \mathrm{GeV}$, where the ISR is expected to have a small effect and thus a kinematic fit is expected to perform well without an additional photon, is used to evaluate whether the addition of such a photon leads to a loss of resolution.

- A subsample with moderate ISR energy $\left(5 \leq E_{\mathrm{ISR}}<30 \mathrm{GeV}\right)$ is used to evaluate a possible bias of the kinematic fit, and whether the addition of a photon removes this bias and increases the fraction of good fits.

- A subsample with large $E_{\mathrm{ISR}} \geq 30 \mathrm{GeV}$ serves to quantify how well the fit with photon works, and whether it has any advantage over a fit where the energy and longitudinal momentum constraints are dropped completely. 


\subsection{Evaluation Method}

In order to investigate the performance of the proposed method, kinematic fits are applied to the four jets in the events from the test sample, comparing the event hypotheses " 4 jets" ( $4 j$ ) and " 4 jets +1 photon" $(4 j+\gamma)$. Both event hypotheses are fitted with five constraints $(5 C$ fit): conservation of energy, conservation of the three momentum components and equal di-jet masses. In addition, the events are fitted also using only the three constraints ( $3 C$-fit) that are not affected by the presence of photon radiation, i.e. conservation of the transverse momentum components and the equal mass constraint.

As discussed in Sect.2.1, both values $p_{\mathrm{z}, \gamma}=0$ and $p_{\mathrm{z}, \gamma}=-p_{\mathrm{z}, \mathrm{miss}}$ are considered as starting values for the photon momentum in the kinematic fit, and the result with the better $\chi^{2}$ is chosen. Values of $\beta=0.1235$ and $E_{\mathrm{ISR}}=225 \mathrm{GeV}$, which is the maximal photon energy that allows $W^{ \pm}$pair production, are used in the photon parametrization Eq. (10). Fig. 2] shows the quantities $z$ and $\eta$ of Eqs. (6) and (7), calculated from $p_{z, \gamma}$ of the most energetic ISR photon in the event. It can be seen that indeed $z$ is distributed uniformly and $\eta$ follows a Gaussian distribution.

The sample used for the performance tests includes the effects of both ISR and beamstrahlung. Since the photon parametrization used here has been derived from the ISR momentum spectrum, tests are first performed that exclude the effect of beamstrahlung. The beamstrahlung is artificially "turned off" by using the total generated energy and momentum of the final state particles, including the ISR photons, in the energy and momentum constraints, rather than the nominal values of $\sum p_{\mathrm{x}, \mathrm{y}, \mathrm{z}}=0, \sum E=\sqrt{s}=500 \mathrm{GeV}$. Alternatively, the constraints are set to these nominal values, so that the combined effects of ISR and beamstrahlung can be studied.

An important indicator for the performance of the various kinematic fits considered is the fraction of good fits, which are defined as those having a fit probability $p>0.001$.

Due to the intrinsic widths of the $W^{ \pm}$and $Z^{0}$ bosons, which are not negligible compared to the detector resolution at an ILD-type detector, the equal-mass constraint is only approximately fulfilled by the four-vectors on generator level and therefore reduces the fraction of good fits.

The equal-mass constraint is applied mainly in order to choose the correct jet pairing. The fit is performed for all three possible jet pairings, and the pairing that results in the best $\chi^{2}$ value is chosen as the correct one under the assumption that the jets stem from either a $W^{+} W^{-}$or a $Z^{0} Z^{0}$ pair. Because of the $W^{ \pm}$and $Z^{0}$ width, the equal-mass constraint leads to an average $\chi^{2}$ contribution that is significantly higher than the value expected for the addition of one constraint, i.e. one degree of freedom. As a consequence, only $55 \%$ of the $3 C$-fits have a fit probability $p>0.001$. A more elaborate treatment of the equal-mass constraint that would increase this fraction is, however, beyond the scope of the present analysis.

Fig. 3 shows the the invariant di-jet masses before and after the kinematic fit for the complete sample, including ISR and beamstrahlung. A clear peak at the $W^{ \pm}$mass is observed, while the much smaller $Z^{0}$ mass peak appears only as an enhancement on the right side of the $W^{ \pm}$mass peak.

Imposing an equal-mass constraint leads to an implicit averaging of the two di-jet masses in each event. Therefore, the average di-jet mass before the fit is compared to the dijet masses after the various kinematic fits in Fig. 3. 
For a quantitative comparison of the different kinematic fits, each mass distribution is fitted with an analytic function. The $W^{ \pm}$mass peak is expected to follow a relativistic Breit-Wigner distribution, folded with a Gaussian distribution that reflects the detector resolution. Here, the mass peaks are fitted with a Voigt function $V_{\sigma, \Gamma}(x)$ [17], which is the convolution of a nonrelativistic Breit-Wigner (Cauchy) distribution of width $\Gamma$ and a Gaussian distribution with an RMS of $\sigma$. Thus the following function is fitted to the histograms in the range $75<m<$ $95 \mathrm{GeV}$ :

$$
f(m)=N \cdot\left(\left(1-f_{Z}\right) \cdot V_{\sigma, \Gamma_{W}}\left(m-m_{\mathrm{W}}\right)+f_{Z} \cdot V_{\sigma, \Gamma_{Z}}\left(m-m_{Z}\right)\right)
$$

The values for the $Z^{0}$ mass $m_{\mathrm{Z}}=91.19 \mathrm{GeV}$ and the decay widths $11 \Gamma_{\mathrm{W}}=2.14 \mathrm{GeV}$ and $\Gamma_{\mathrm{Z}}=2.50 \mathrm{GeV}$ are fixed to their literature values [18]. The same Gaussian width $\sigma$, reflecting the detector resolution, is used for the $W^{ \pm}$and $Z^{0}$ mass peaks. $N$ corresponds to the number of histogram entries and $f_{Z}$ to the fraction of $Z$-pair events. However, because the symmetric Voigt function does not describe the asymmetry of a relativistic Breit-Wigner distribution correctly, $f_{Z}$ is not an accurate estimate of the fraction of $Z^{0}$ events in the sample.

The parameters of interest are the Gaussian width $\sigma$ and the difference $\Delta m_{\mathrm{W}}=m_{\mathrm{W}}-m_{\mathrm{W}}^{\text {gen }}$ between the fitted $W^{ \pm}$mass $m_{\mathrm{W}}$ and the input $W^{ \pm}$mass $m_{\mathrm{W}}^{\text {gen }}$.

If large amounts of energy are missing, the fitted jet energies have to be larger than the measured ones to fulfill energy conservation. Consequently, di-jet masses are shifted to higher values and thus a larger $\Delta m_{\mathrm{W}}$ is obtained. Due to the imperfections of the lineshape fit, a nonzero value of $\Delta m_{\mathrm{W}}$ is to be expected, for which a correction would be applied in a real analysis. However, if this mass shift depends on the amount of energy from ISR and beamstrahlung, it leads to a broadening of the signal and thus a loss of resolution; in addition, systematic uncertainties arise from the description of the ISR and in particular the beamstrahlung energy spectrum. Therefore, a mass shift that is independent of the amount of energy lost to ISR and beamstrahlung is desirable.

\subsection{Results}

Tab. 1 summarizes the results of our tests. It lists the fraction of good fits, the mass shift and the width of the Gaussian part of the Voigt function for the complete sample, as well as the three subsamples with different amounts of missing energy due to ISR photons. The results are given for the average of the di-jet masses before a kinematic fit, using the $3 C$ jet pairing, as well as the di-jet mass after applying a $3 C$ fit or a $5 C$ fit without or with an ISR photon. The results are reported for the case where the effect from beamstrahlung has been excluded by adjusting the energy and momentum constraints (cf. Sect. 3.2), and for the realistic case where effects from ISR and beamstrahlung are fully taken into account.

\section{Results with ISR only}

A comparison of the fit results demonstrates the gain in resolution achieved by kinematic fitting: The Gaussian $\sigma$, which corresponds to the di-jet mass resolution, is $\sigma=2.1 \mathrm{GeV}$ for the

\footnotetext{
${ }^{1}$ The average of two independent random numbers distributed according to a Breit-Wigner of width $\Gamma$ follows a Breit-Wigner distribution of the same width $\Gamma$.
} 


\begin{tabular}{|c|c|c|c|c|c|c|c|}
\hline \multirow{2}{*}{$\begin{array}{l}\text { Subsample } \\
\text { (Fraction) }\end{array}$} & \multirow{2}{*}{$\begin{array}{l}\text { Constraints, } \\
\text { Hypothesis }\end{array}$} & \multicolumn{3}{|c|}{ ISR only } & \multicolumn{3}{|c|}{ Full Photon Spectrum } \\
\hline & & $\begin{array}{l}\text { Good } \\
\text { fits }[\%]\end{array}$ & $\begin{array}{l}\Delta m_{\mathrm{W}} \\
{[\mathrm{GeV}]}\end{array}$ & $\begin{array}{c}\sigma_{\mathrm{W}} \\
{[\mathrm{GeV}]}\end{array}$ & $\begin{array}{l}\text { Good } \\
\text { fits }[\%]\end{array}$ & $\begin{array}{l}\Delta m_{\mathrm{W}} \\
{[\mathrm{GeV}]}\end{array}$ & $\begin{array}{c}\sigma_{\mathrm{W}} \\
{[\mathrm{GeV}]}\end{array}$ \\
\hline \multirow{4}{*}{$\begin{array}{l}\text { All events } \\
(100 \%)\end{array}$} & - - & $55 \%$ & +0.78 & 2.05 & $55 \%$ & +0.78 & 2.05 \\
\hline & $3 C, 4 j$ & $55 \%$ & +0.82 & 2.06 & $55 \%$ & +0.82 & 2.06 \\
\hline & $5 C, 4 j$ & $42 \%$ & +0.67 & 1.21 & $31 \%$ & +0.91 & 1.30 \\
\hline & $5 C, 4 j+\gamma$ & $54 \%$ & +0.53 & 1.25 & $52 \%$ & +0.75 & 1.35 \\
\hline \multirow{4}{*}{$\begin{array}{l}E_{\mathrm{ISR}}<5 \mathrm{GeV} \\
(75 \%)\end{array}$} & - & $56 \%$ & +0.80 & 2.04 & $56 \%$ & +0.80 & 2.04 \\
\hline & $3 C, 4 j$ & $56 \%$ & +0.85 & 2.06 & $56 \%$ & +0.85 & 2.06 \\
\hline & $5 C, 4 j$ & $53 \%$ & +0.63 & 1.19 & $40 \%$ & +0.86 & 1.27 \\
\hline & $5 C, 4 j+\gamma$ & $55 \%$ & +0.49 & 1.24 & $54 \%$ & +0.69 & 1.31 \\
\hline \multirow{4}{*}{$\begin{array}{l}5 \leq E_{\mathrm{ISR}}<30 \mathrm{GeV} \\
(11 \%)\end{array}$} & - & $54 \%$ & +0.79 & 2.07 & $54 \%$ & +0.79 & 2.07 \\
\hline & $3 C, 4 j$ & $54 \%$ & +0.84 & 2.08 & $54 \%$ & +0.84 & 2.08 \\
\hline & $5 C, 4 j$ & $15 \%$ & +1.68 & 1.25 & $12 \%$ & +2.19 & 1.29 \\
\hline & $5 C, 4 j+\gamma$ & $53 \%$ & +0.71 & 1.27 & $50 \%$ & +1.07 & 1.51 \\
\hline \multirow{4}{*}{$\begin{array}{l}E_{\mathrm{ISR}} \geq 30 \mathrm{GeV} \\
(13 \%)\end{array}$} & - & $53 \%$ & +0.59 & 1.99 & $53 \%$ & +0.59 & 1.99 \\
\hline & $3 C, 4 j$ & $53 \%$ & +0.66 & 1.99 & $53 \%$ & +0.66 & 1.99 \\
\hline & $5 C, 4 j$ & $0 \%$ & - & - & $0 \%$ & - & - \\
\hline & $5 C, 4 j+\gamma$ & $47 \%$ & +0.64 & 1.21 & $42 \%$ & +0.91 & 1.38 \\
\hline
\end{tabular}

Table 1: Results of kinematic fits under various conditions. "ISR only" refers to the case where the effect of beamstrahlung and beam energy spread is removed from the fit as explained in the text, while "Full Photon Spectrum" includes these effects. For each fit variation, the fraction of good fits with fit probability $p>0.001$, the difference $\Delta m_{\mathrm{W}}$ between the fitted and generated $\mathrm{W}$ mass of $m_{\mathrm{W}}^{\text {gen }}=80.419 \mathrm{GeV}$, and the width of the Gaussian part of the Voigt function is given. The rows refer to the results from averaging the measured di-jet masses without a fit for events where the $3 \mathrm{C}$ fit converges, the $3 \mathrm{C}$ fit with only transverse momentum and equalmass constraint, the $5 \mathrm{C}$ fit under a four jet hypothesis with longitudinal momentum and energy constraints in addition, and the 5C fit with an additional ISR photon fit object. The subsamples are distinguished by the total energy $E_{\mathrm{ISR}}$ of ISR photons, excluding beamstrahlung.

average of the two di-jet masses without a kinematic fit and improves to $\sigma=1.3 \mathrm{GeV}$ if a kinematic fit with five constraints is used. A fit with only three constraints does not improve the resolution compared to the simple averaging of the unfitted di-jet masses.

The fit with five constraints and no ISR photon cannot be applied to the subsample with $E_{\mathrm{ISR}} \geq 30 \mathrm{GeV}$, because fit probabilities above the cut of $p=0.001$ are essentially never achieved due to the missing energy and momentum that are are too large to be accomodated by the experimental resolution of a few GeV. Therefore this subsample, which contains $13 \%$ of all events, cannot be used for an analysis. The $5 C$ fit with an ISR photon, on the other hand, achieves almost the same performance for the two subsamples with $E_{\mathrm{ISR}} \geq 30 \mathrm{GeV}$ and $E_{\mathrm{ISR}}<5 \mathrm{GeV}$ in terms of the fraction of good fits ( $47 \%$ vs. $55 \%$ ) as well as in resolution $(\sigma=$ $1.21 \mathrm{GeV}$ vs. $1.24 \mathrm{GeV})$ with only a small additional bias in the $\mathrm{W}$ mass $\left(\Delta m_{\mathrm{W}}=0.64 \mathrm{GeV}\right.$ vs. $0.49 \mathrm{GeV})$.

The sample with moderate ISR energy $5 \leq E_{\mathrm{ISR}}<30 \mathrm{GeV}$, which comprises $11 \%$ of the events, demonstrates that the $5 C$ fit without the inclusion of an ISR photon tends to develop 
a mass bias. This is because the energy carried away by the photon is falsely attributed to the final state jets, which increases their energy and thus the invariant mass: The mass bias increases from $\Delta m_{\mathrm{W}}=+0.63 \mathrm{GeV}$ to $+1.68 \mathrm{GeV}$. At the same time, only $15 \%$ of the events yield a good $5 C$ fit under the $4 j$ hypothesis. In contrast, the $4 j+\gamma$ hypothesis shows the same performance in terms of fraction of good fit, mass shift and resolution as for the sample with small missing energy.

The fact that for all fit hypotheses only about half of the events have reasonable fit probabilities $p>0.001$ can be mostly attributed to the equal-mass constraint: The resolution for the difference of the di-jet masses is approxiately $4.1 \mathrm{GeV}$ (twice the resolution for the di-jet mass average for the unfitted jets), which is of similar size as the broadening 2 of $4.3 \mathrm{GeV}$ due to the intrinsic $\mathrm{W}$ width. This indicates that in a real analysis the naïve equal-mass constraint has to be modified to take the natural $W$ width into account. Other factors that reduce the fraction of successful fits are events from processes other than $W / Z$ boson pair production and the fact that the jet error parametrization employed in this analysis does not include the effects of parton showering.

\section{Results with ISR and beamstrahlung}

The right-hand side of Tab. 1 shows the results for the case where the effect of both, ISR and beamstrahlung, is considered. Because the three subsamples are defined on the basis of the ISR energy only, the same amount of beamstrahlung is present in each of them. A comparison with the case where only the effect from ISR is considered, demonstrates that the photon momentum parametrization Eq. (10) derived from the ISR momentum spectrum also works quite well in the presence of beamstrahlung, at least at the level of beamstrahlung that is expected for the nominal ILC parameter set.

Since beamstrahlung in the Monte Carlo simulation used for this analysis is simulated solely through a variation of the energy of the incoming leptons, no transverse momentum is carried by the beamstrahlung. Therefore the results for the $3 C$ fit and the di-jet masses calculated without a kinematic fit do not change when beamstrahlung effects are considered.

The performance of the $5 C$ fit under the $4 j$ hypothesis is significantly reduced when beamstrahlung effects are considered due to the larger amount of missing energy. Overall, the fraction of good fits goes down from $42 \%$ to $31 \%$. For the subsample with less than $5 \mathrm{GeV}$ of ISR energy it is reduced from $53 \%$ to $40 \%$. At the same time, the $W^{ \pm}$mass shift increases by approximately $0.2 \mathrm{GeV}$ for the whole sample. For the subsample with medium $E_{\mathrm{ISR}}$, however, the mass shift increases from $+1.68 \mathrm{GeV}$ to $+2.19 \mathrm{GeV}$.

On the other hand, with the $4 j+\gamma$ hypothesis, the $5 C$ fit performance is much less affected by beamstrahlung effects: The fraction of good fits stays almost constant, and the $\sigma$ of the Gaussian width of the mass peak increases only moderately, from $1.25 \mathrm{GeV}$ to $1.35 \mathrm{GeV}$ for the complete sample. The mass shift increases by approximately $0.2 \mathrm{GeV}$ for the full sample, which is similar to the $4 j$ hypothesis. However, for the subsample with $5<E_{\text {ISR }}<30 \mathrm{GeV}$ the mass shift is significantly reduced from +2.19 to $+1.07 \mathrm{GeV}$ by the inclusion of the photon

\footnotetext{
${ }^{2}$ The difference of two Breit-Wigner distributed random numbers follows itself a Breit-Wigner function with a width that is the sum of the two individual widths.
} 
in the fit. The increase of the mass shift with respect to the ISR only case indicates that the $4 j+\gamma$ hypothesis cannot fully accomodate beamstrahlung effects, because typically both beam particles radiate off significant energy. This may necessitate the inclusion of a second photon in the fit.

As a final check, Fig. 4 shows the fitted longitudinal momentum $p_{z, \gamma}$ of the photon versus the generated $p_{\mathrm{z}, \gamma}^{\mathrm{gen}}$ of the most energetic ISR+beamstrahlung photon pair in the event, where the momenta of the ISR and beamstrahlung photons with either positive or negative $p_{\mathrm{z}}$ are added. It can be seen that the fitted photon momentum $p_{\mathrm{z}, \gamma}$ corresponds quite well to the true momentum, without any visible bias. In particular, the fact that the photon is treated as having a measured $p_{\mathrm{z}, \gamma}=0$ does not lead to a large bias towards small values of $p_{\mathrm{z}, \gamma}$. This is explained by the fact that the function $p_{\mathrm{z}, \gamma}(\eta)$ of Eq. (10) rises very rapidly, as discussed in Sect. 2.1.

The right side of Fig 4 shows the difference $\Delta p_{\mathrm{z}, \gamma}=\operatorname{sign}\left(p_{\mathrm{z}, \gamma}\right) \cdot\left(p_{\mathrm{z}, \gamma}-p_{\mathrm{z}, \gamma}^{\mathrm{gen}}\right)$. The mean $\left\langle\Delta p_{\mathrm{z}, \gamma}\right\rangle=-0.32 \mathrm{GeV}$ is small, and negative, showing that the reconstructed $\left|p_{\mathrm{z}, \gamma}\right|$ is slightly smaller on average than the generated one, as expected, but that this bias is indeed quite small. The resolution for $p_{\mathrm{z}, \gamma}$ is found to be $3.25 \mathrm{GeV}$.

\section{Summary and Conclusions}

In this paper a method is proposed to take the effect of ISR into account in kinematic fits by introducing a photon that is treated as if its measured momentum were zero. The longitudinal momentum $p_{\mathrm{z}, \gamma}$ is expressed as a function $p_{\mathrm{z}, \gamma}(\eta)$ of the parameter $\eta$ such that the true value of $\eta$ follows a normal distribution with zero mean and unit standard deviation.

The performance of this method is evaluated using a sample of $e^{+} e^{-} \rightarrow u \bar{d} d \bar{u}$ events, which is dominated by $W^{+} W^{-}$pair production, at $\sqrt{s}=500 \mathrm{GeV}$. The sample includes the effects from ISR and beamstrahlung. It is fully simulated and reconstructed, using the simulation for the ILD detector at the ILC. A $5 C$ kinematic fit with energy and momentum conservation constraints and an equal-mass constraint is applied, and the results for the fit hypothesis with four jets and a photon are compared to three alternatives: a $5 C$ fit with a conventional four jet hypothesis, a $3 C$ fit where the energy and longitudinal momentum constraints are dropped, and the results obtained without a kinematic fit.

The $5 C$ fit with the new $4 j+\gamma$ hypothesis performs as well as a $5 C$ fit with a $4 j$ hypothesis in terms of resolution, while a $3 C$ is significantly worse and does not yield any improvement over a mass reconstruction without any kinematic fit.

For events with significant energy from ISR photons $\left(5<E_{\mathrm{ISR}}<30 \mathrm{GeV}\right)$, the fraction of good fits with a fit probability $p>0.001$ drops from $40 \%$ to $12 \%$ for a $5 C$ fit without a photon, and goes to zero for $E_{\mathrm{ISR}}>30 \mathrm{GeV}$. In addition, as the missing energy is distributed to the jets by such a fit, a shift of the reconstructed di-jet masses towards larger values is observed.

Both problems are solved by the new $4 j+\gamma$ hypothesis: even for large values of $E_{\mathrm{ISR}}>$ $30 \mathrm{GeV}$, the fraction of good fits and the di-jet mass resolution are similar to the values obtained at $E_{\mathrm{ISR}}<5 \mathrm{GeV}$, while the mass shift remains small. 
In short, under the $4 j+\gamma$ hypothesis, a $5 C$ fit achieves the same resolution as with a conventional $4 j$ fit hypothesis, but independent of the amount of ISR energy, without developing a mass bias, and with a similar fraction of good fits as a $3 C$ fit.

Although the parametrization $p_{z, \gamma}(\eta)$ was developed using the momentum spectrum of ISR photons, the method also performs well in the presence of beamstrahlung, at least at the moderate level expected for the nominal parameter set of the ILC.

In a future development the parametrization could be adapted to include beamstrahlung effects. This may be necessary in scenarios with enhanced beamstrahlung, such as the "low power" parameter set proposed for the ILC, or at CLIC. We expect that under such conditions the addition of a second photon in the fit would become necessary in order to take into account the energy loss suffered by both beam particles.

\section{Acknowledgements}

We would like to thank the ILD simulation production team, in particular F. Gaede, S. Aplin, J. Engels and I. Marchesini, for the production of the samples of events used in this work, and T. Barklow for producing the generated input files.

We acknowledge the support of the DFG through the SFB (grant SFB 676/1-2006) and the Emmy-Noether program (grant LI-1560/1-1).

\section{References}

[1] M. A. Thomson, Eur. Phys. J. C 33 (2004) S689.

[2] J. Brau, Y. Okada and N. Walker (eds.) [ILC Collaboration], "ILC Reference Design Report Volume 1 - Executive Summary," arXiv:0712.1950 [physics.acc-ph] (2007); G. Aarons et al. [ILC Collaboration], "ILC Reference Design Report Volume 2 - Physics at the ILC," arXiv:0709.1893 [hep-ph] (2007);

N. Phinney, N. Toge and N. Walker (eds.), "ILC Reference Design Report Volume 3 Accelerator," arXiv:0712.2361 [physics.acc-ph] (2007);

T. Behnke, C. Damerell, J. Jaros and A. Miyamoto (eds.) [ILC Collaboration], "ILC Reference Design Report Volume 4 — Detectors," arXiv:0712.2356 [physics.ins-det] (2007).

[3] R. W. Assmann et al., "A $3 \mathrm{TeV} e^{+} e^{-}$linear collider based on CLIC technology," CERN2000-008, SLAC-REPRINT-2000-096 (2000);

H. Braun et al. [CLIC Study Team Collaboration], "CLIC 2008 parameters," CLICNOTE-764 (2008).

[4] B. List and J. List, "MarlinKinfit: An object-oriented kinematic fitting package," LCTOOL-2009-001 (2009), http://www-flc.desy.de/lcnotes.

[5] F. A. Berends, R. Kleiss and S. Jadach, Nucl. Phys. B 202 (1982) 63;

idem, Comput. Phys. Commun. 29 (1983) 185. 
[6] K. Yokoya and P. Chen, in: M. Dienes, M. Month and S. Turner, "Frontiers of particle beams: intensity limitations: proceedings," Springer, Lecture notes in physics 400 (1992) 415 [KEK-PREPRINT-91-2];

P. Chen, Phys. Rev. D 46 (1992) 1186;

P. Chen, T. L. Barklow and M. E. Peskin, Phys. Rev. D 49 (1994) 3209 [arXiv:hep-ph/9305247];

M. E. Peskin, “Consistent Yokoya-Chen approximation to beamstrahlung," SLAC-TN-04032, LCC-0010 (1999).

[7] ILD Concept Group, “The International Large Detector — Letter of Intent,” DESY-200987 (2009).

[8] M. Beckmann, "Verbesserung der $W W / Z Z$-Unterscheidung am ILC durch Berücksichtigung von Photonabstrahlung in kinematischen Fits," Diploma thesis, Leibniz University of Hannover (2009), DESY-THESIS-2010-014.

[9] W. Kilian, T. Ohl and J. Reuter, "WHIZARD: Simulating multi-particle processes at LHC and ILC," arXiv:0708.4233 [hep-ph] (2007).

[10] D. Schulte, "Beam-beam simulations with GUINEA-PIG," CERN-CLIC-NOTE-387 (1998).

[11] A. Seryi, "Power saving optimization for Linear Collider interaction region parameters," SLAC-PUB-13767 (2009);

idem, "IP and bunch parameters for ILC," http://ilcagenda.linearcollider.org/ contributionDisplay ·py? contribId=113\&session Id=9\& conf Id=2432 (2008);

idem, "ILC Beam parameters including $250 \mathrm{GeV}$ ILC," http://ilcphys.kek.jp/soft/ ILCBeam/BeamParameter.html (2008).

[12] "MOKKA: A detailed Geant4 simulation for the International Linear Collider detectors, version 06-07-p01,"http://polzope.in2p3.fr:8081/MOKKA

[13] O. Wendt, F. Gaede and T. Krämer, Pramana 69 (2007) 1109 [arXiv:physics/0702171].

[14] M. A. Thomson, AIP Conf. Proc. 896 (2007) 215.

[15] S. Bethke, Z. Kunszt, D. E. Soper and W. J. Stirling, Nucl. Phys. B 370 (1992) 310 [Erratum-ibid. B 523 (1998) 681].

[16] D. Ward and W. Yan, " $B r(H \rightarrow c \bar{c})$ from $Z H \rightarrow q \bar{q} c \bar{c} @ 250 \mathrm{GeV}$," http://www.ilcild.org/groups/optimization/loi-physics-analyses-1/ higgs-branching-ratios-at-250-gev-zh-qqbb-qqcc/zh.pdf/view (2009).

[17] B.H. Armstrong, J. Quant. Spectrosc. Radiat. Trans. 7 (1967) 61.

[18] C. Amsler et al. [Particle Data Group], Phys. Lett. B 667 (2008) 1. 

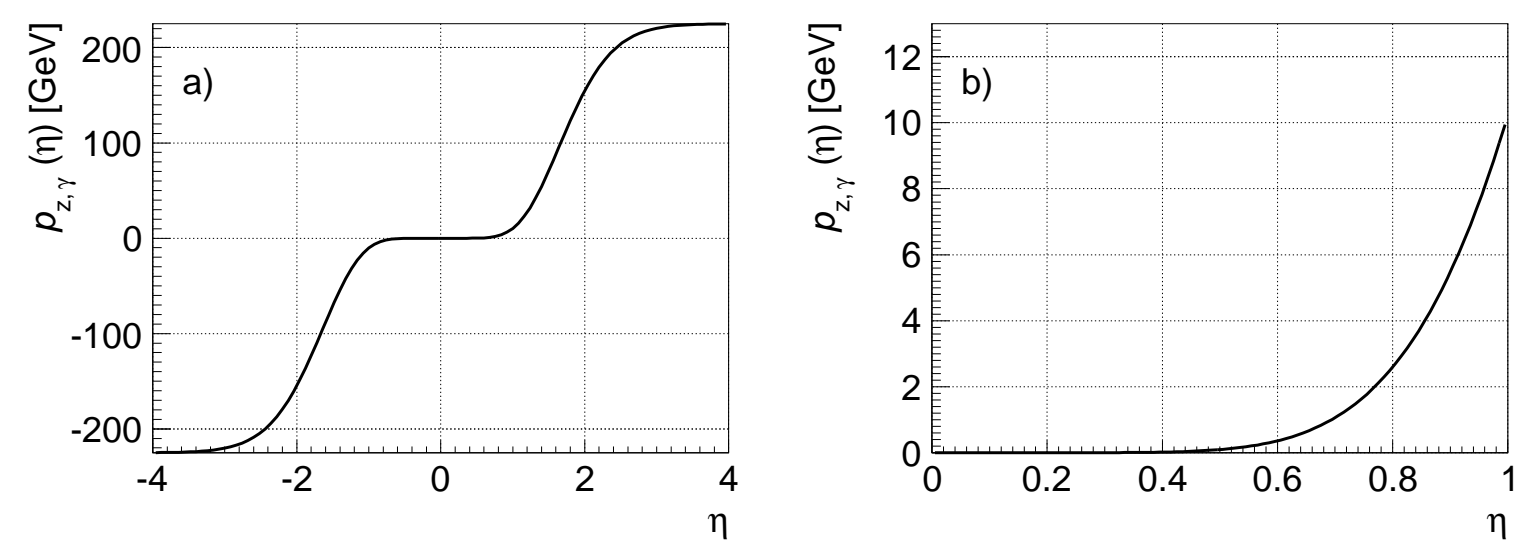

Figure 1: The photon's $z$-momentum $p_{\mathrm{z}, \gamma}$ as a function of the fit parameter $\eta$, as given by Eq. (10), for $E_{\max }=225 \mathrm{GeV}$ and $\beta=0.1235$ in the range $|\eta|<4$ (a) and $0<\eta<1$ (b).
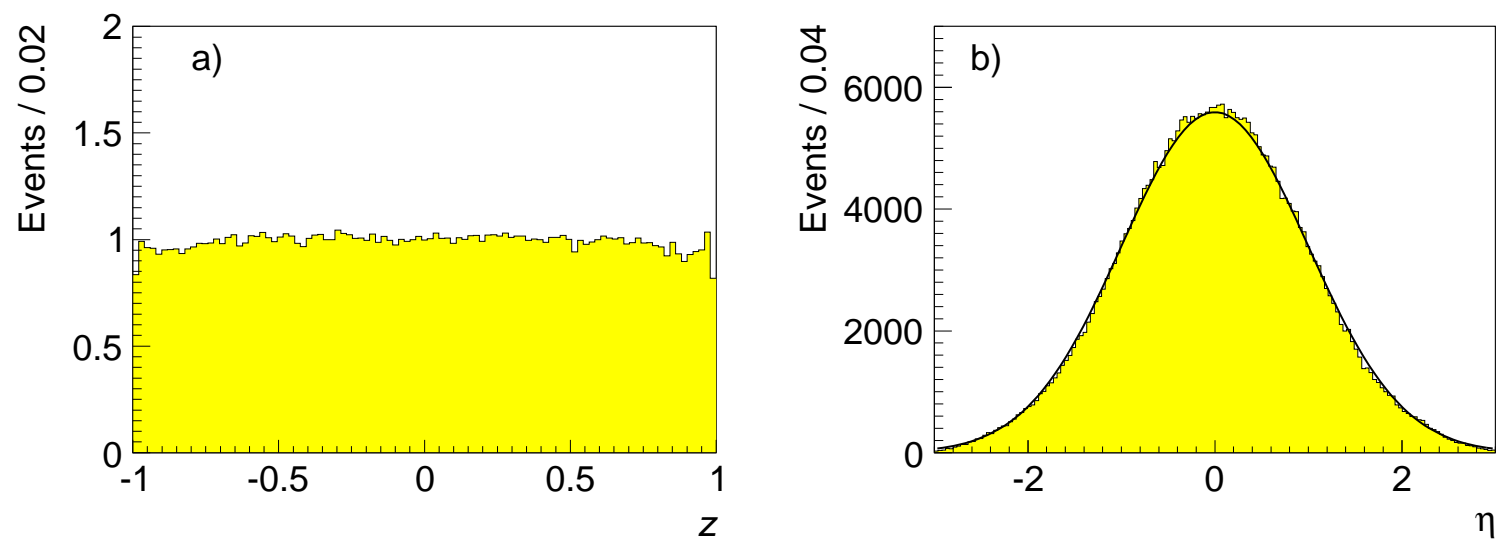

Figure 2: The parameters $z$ (a) of Eq. (6) and $\eta$ (b) of Eq. (7), calculated from the longitudinal momentum $p_{\mathrm{z}, \gamma}$ of the most energetic ISR photon in the Monte Carlo sample described in the text, using $E_{\max }=225 \mathrm{GeV}$ and $\beta=0.1235$. $z$ is expected to be uniformely distributed in $-1<z<1$, and $\eta$ should follow a Gaussian distribution with zero mean and unit standard deviation, which is shown for comparison in the plot. 

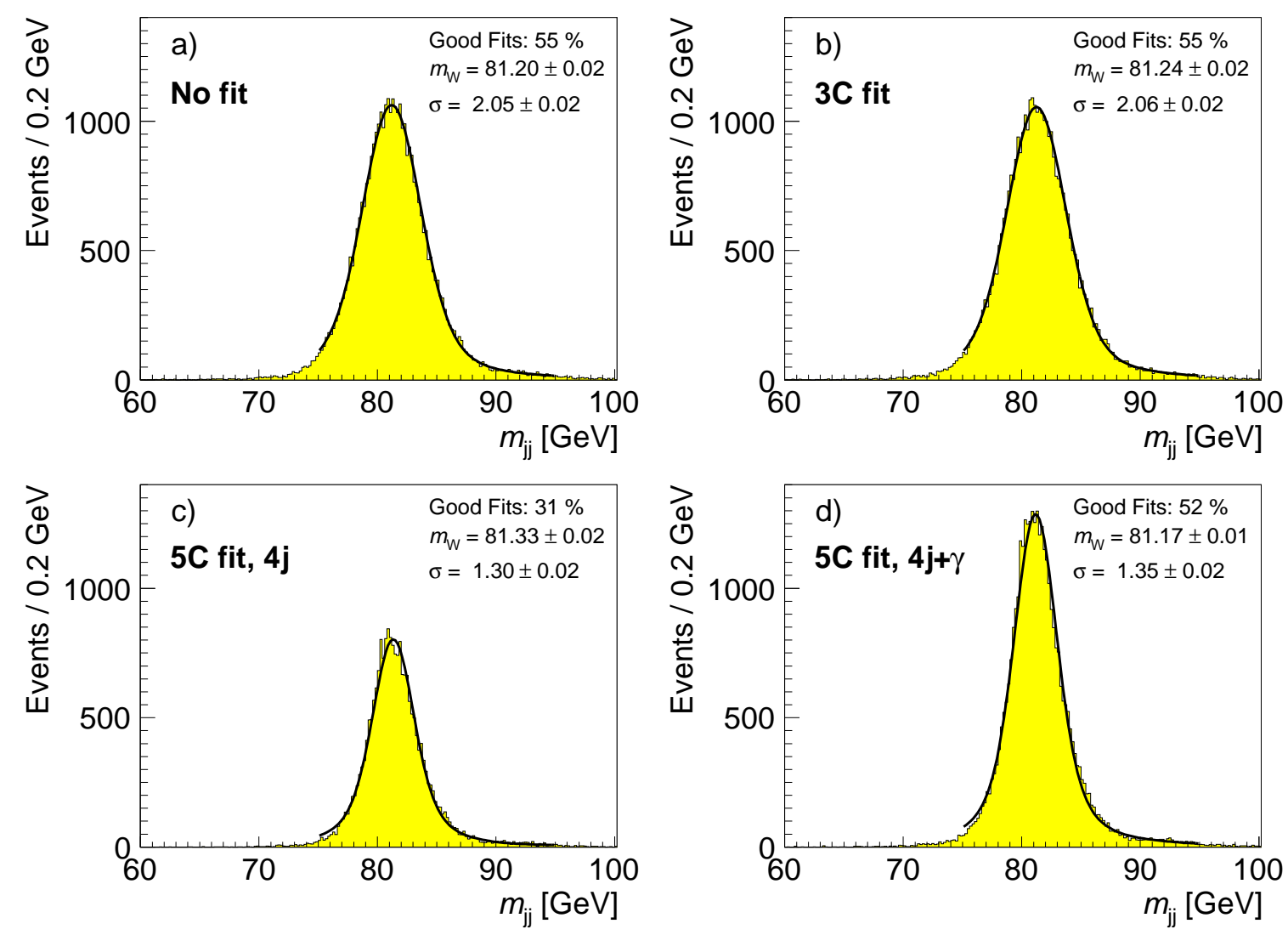

Figure 3: Invariant di-jet masses $m_{\mathrm{jj}}$ for the Monte Carlo sample described in the text: a) the average of the two di-jet masses calculated from the measured four-vectors, using the jet pairing from the $3 C$ fit sample; b) $m_{\mathrm{jj}}$ after application of the $3 C$ fit; c) $m_{\mathrm{jj}}$ for the $5 C$ fit under a $4 j$ hypothesis; d) $m_{\mathrm{jj}}$ for the $5 C$ fit under a $4 j+\gamma$ hypothesis.
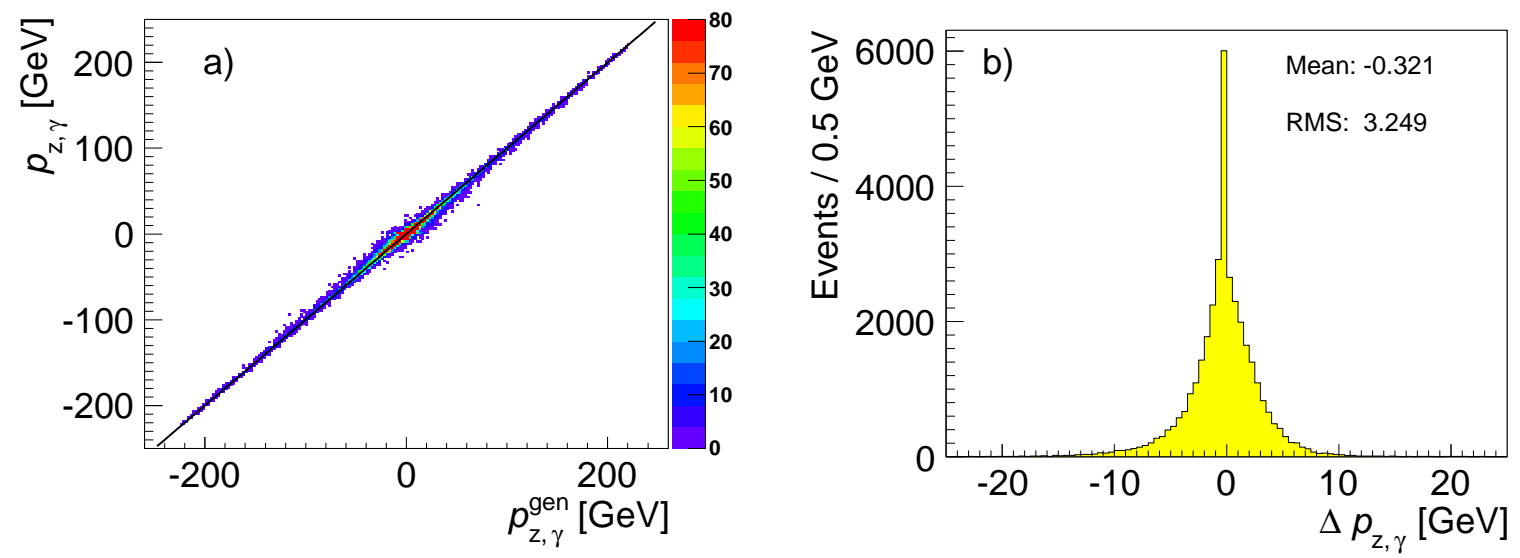

Figure 4: Fitted photon momentum $p_{\mathrm{z}, \gamma}$ plotted against the true momentum $p_{\mathrm{z}, \gamma}^{\mathrm{gen}}$ of the most energetic ISR+beamstrahlung photon combination in the event (a), and the difference $\Delta p_{\mathrm{z}, \gamma}=$ $\operatorname{sign}\left(p_{\mathrm{z}, \gamma}\right) \cdot\left(p_{\mathrm{z}, \gamma}-p_{\mathrm{z}, \gamma}^{\mathrm{gen}}\right)(\mathrm{b})$. 